\title{
Optical coherence elastography assessment of corneal viscoelasticity with a modified Rayleigh-Lamb wave model
}

Zhaolong $\mathrm{Han}^{\mathrm{a}, \uparrow}$, Jiasong $\mathrm{Li}^{\mathrm{a}, \dagger}$, Manmohan Singh ${ }^{\mathrm{a}, \dagger}$, Chen $\mathrm{Wu}^{\mathrm{a}}$, Chih-hao Liu ${ }^{\mathrm{a}}$, Raksha Raghunathan $^{\mathrm{a}}$, Salavat R. Aglyamov ${ }^{\mathrm{b}}$, Srilatha Vantipalli ${ }^{\mathrm{c}}$, Michael D. Twa ${ }^{\mathrm{d}}$, and Kirill V. $\operatorname{Larin}^{\mathrm{a}, \mathrm{e}, \mathrm{f}, *}$

${ }^{a}$ Department of Biomedical Engineering, University of Houston, Houston, TX, 77204

${ }^{b}$ Department of Biomedical Engineering, University of Texas at Austin, Austin, TX, 78712

${ }^{\mathrm{c} C o l l e g e ~ o f ~ O p t o m e t r y, ~ U n i v e r s i t y ~ o f ~ H o u s t o n, ~ H o u s t o n, ~ T X, ~} 77204$

${ }^{\mathrm{d}}$ School of Optometry, University of Alabama at Birmingham, Birmingham, AL, 35233

${ }^{\mathrm{e}}$ Interdisciplinary Laboratory of Biophotonics, Tomsk State University, Tomsk, Russia

${ }^{\mathrm{f}}$ Department of Molecular Physiology and Biophysics, Baylor College of Medicine, Houston, TX, 77030

${ }^{\dagger}$ Contribute equally to the present work

*Address all correspondence to:

Kirill V. Larin

University of Houston Department of Biomedical Engineering 3517 Cullen Blvd, Room 2027

Houston, TX 77204

klarin@central.uh.edu

UV-A/riboflavin corneal collagen crosslinking: CXL

Optical coherence elastography: OCE

Phase-stabilized swept source optical coherence tomography: PhS-SSOCT

Intraocular pressure: IOP

Rayleigh-Lamb frequency equation: RLFE

Modified Rayleigh-Lamb frequency equation: mRLFE 


\begin{abstract}
The biomechanical properties of the cornea play a critical role in forming vision. Diseases such as keratoconus can structurally degenerate the cornea causing a pathological loss in visual acuity. UV-A/riboflavin corneal collagen crosslinking (CXL) is a clinically available treatment to stiffen the cornea and restore its healthy shape and function. However, current CXL techniques do not account for pre-existing biomechanical properties of the cornea nor the effects of the CXL treatment itself. In addition to the inherent corneal structure, the intraocular pressure (IOP) can also dramatically affect the measured biomechanical properties of the cornea. In this work, we present the details and development of a modified Rayleigh-Lamb frequency equation model for quantifying corneal biomechanical properties. After comparison with finite element modeling, the model was utilized to quantify the viscoelasticity of in situ porcine corneas in the whole eye-globe configuration before and after CXL based on noncontact optical coherence elastography measurements. Moreover, the viscoelasticity of the untreated and CXL-treated eyes was quantified at various IOPs. The results showed that the stiffness of the cornea increased after CXL and that corneal stiffness is close to linear as a function of IOP. These results show that the modified Rayleigh-Lamb wave model can provide an accurate assessment of corneal viscoelasticity, which could be used for customized CXL therapies.
\end{abstract}

\title{
Keywords:
}

modified Rayleigh-Lamb frequency equation, optical coherence elastography, viscoelasticity, cornea, crosslinking, intraocular pressure 


\section{Introduction}

The cornea is a critical component of our visual system because it provides approximately $2 / 3$ of the total refracting power of the eye (Ruberti et al., 2011). Moreover, the biomechanical properties of the cornea are inherently tied to its health due to its shape and function. Diseases such as keratoconus can pathologically degenerate the cornea, and its shape causing degraded vision or even complete vision loss (Gore et al., 2014; Ortiz-Toquero et al., 2015; Sahebjada et al., 2014). Therapeutic interventions such as LASIK surgeries or UV-A/riboflavin corneal collagen crosslinking (CXL) can also alter corneal biomechanical properties (Randleman et al., 2015; Richoz et al., 2013; Wollensak et al., 2003a). Therefore, assessing corneal biomechanical properties can provide crucial information for assessing corneal health and integrity as well as evaluating the efficacy of therapeutic interventions, particularly those aimed at altering corneal biomechanical properties such as CXL.

CXL is a rapidly emerging clinical treatment for keratoconus that stiffens the cornea and prevents or minimizes further degeneration (Wollensak et al., 2003a; Zhang et al., 2015). While CXL has been proposed as a treatment for other ocular pathologies such as keratitis (Iseli et al., 2008), its main use remains the treatment of keratoconus. In addition to the traditional "Dresden" CXL protocol (Wollensak et al., 2003a), a variety of CXL techniques have emerged, varying from accelerated methods ( $\mathrm{Ng}$ et al., 2016) to pulsed techniques (Mazzotta et al., 2014) to spatially varying methods for customized therapies (Seiler et al., 2016). However, the current clinical protocol still remains a blanket treatment and does not account for pre-existing biomechanical properties nor the effects of the treatment itself. In addition to changes in the 
intrinsic corneal structure, the intraocular pressure (IOP) can affect measurement of corneal biomechanical properties and vice versa (Bao et al., 2015; Liu and He, 2009). Our previous work has shown that although the elasticity as measured by OCE may be the same due to an elevated IOP or CXL, the damping characteristics are different (Li et al., 2014a). However, the damping was quantified by a relativistic metric that compared the amplitude damping of an air-pulse induced elastic wave rather than quantitative material properties such as viscosity.

Therefore, there is a direct need for a technique that can accurately and robustly measure the biomechanical properties of the cornea. The Ocular Response Analyzer (ORA) can provide the corneal hysteresis, which is related to the cornea damping ability by emitting a strong air puff to the corneal surface (Tao et al., 2013). The CorVis ST is able to image dynamic corneal movement, which is also stimulated by a similar large volume air puff (Han et al., 2014). However, the measurements provided by these approaches can only provide information of the whole cornea due to the large volume of air, and there is conflicting evidence in the literature on their ability to detect changes in corneal biomechanical properties due to pathologies or therapeutic interventions (Bak-Nielsen et al., 2014; Gkika et al., 2012; Goldich et al., 2009; Greenstein et al., 2012). Brillouin microscopy is an emerging technique that can map the 3D distribution of elasticity characteristics by investigating the Brillouin frequency shift within the corneal tissue (Scarcelli et al., 2012). However, obtaining quantitative biomechanical parameters such as Young's modulus and viscosity from the Brillouin shift is still a challenge.

Elastography has emerged as an effective nondestructive technique for quantifying tissue biomechanical properties. Since development in the 1990s, ultrasound elastography (Ophir et al., 1991) and magnetic resonance elastography (Muthupillai et al., 1995) have become clinically available tools for detecting pathologies such as thyroid malignancies (Azizi et al., 2015), breast 
cancer (Aglyamov et al., 2012; Faruk et al., 2015), and hepatic fibrosis (Venkatesh et al., 2013). However, these techniques require a relatively large displacement to produce a detectable signal, have limited spatial resolution, and require contact with the tissue, all of which restrict their use on small and thin samples such as the cornea.

Optical coherence tomography (OCT) based elastography, which is termed optical coherence elastography (OCE) (Schmitt, 1998; Wang and Larin, 2015), can overcome the aforementioned limitations due to the micrometer-scale resolution of OCT (Huang et al., 1991). Moreover, analyzing the phase of the complex OCT signal has enabled sub-nanometer displacement sensitivity (Sticker et al., 2001). Similar to traditional elastographic techniques, OCE utilizes its parent imaging modality of OCT to detect externally induced displacements within tissue. Due to the high resolution and noncontact nature of OCT, OCE is particularly well suited for assessing the biomechanical properties of the cornea (Ford et al., 2011; Ford et al., 2014; Han et al., 2015b; Li et al., 2014a; Li et al., 2013; Li et al., 2014b; Qu et al., 2016; Singh et al., 2016a; Singh et al., 2016b; Singh et al., 2016c; Singh et al., 2015; Twa et al., 2014; Wang and Larin, 2014a, b).

However, a common issue in reconstructing tissue biomechanical properties is the application of appropriate models. Simple models such as the shear wave equation and surface wave equation have been used previously to quantify corneal biomechanical properties ( $\mathrm{Li}$ et al., 2013; Singh et al., 2016c; Singh et al., 2015). However, assumptions about sample geometry, such as an infinite thickness, are not directly applicable to the cornea. The thickness of the cornea can change due to hydration (Hedbys and Mishima, 1966), intraocular pressure (Bao et al., 2015; Liu and Roberts, 2005), various pathologies (Ambrosio et al., 2006), and/or therapeutic interventions (Rosenblat and Hersh, 2016). We have shown that a change in corneal thickness can affect 
elastographic measurements (Han et al., 2015b), and thus, there is a need for a mechanical model that can incorporate the thickness of the cornea.

In our previous work (Han et al., 2015a), the viscoelasticity of an in situ porcine cornea was quantified by utilizing OCE measurements and a modified Rayleigh-Lamb frequency equation (mRLFE) model. However, measurements were only made at a single IOP of a single in situ untreated porcine cornea. In this paper, we detail the development of the mRLFE model and have utilized the mRLFE to fit dispersion curves obtained from air-pulse OCE measurements of in situ porcine corneas in the whole eye-globe configuration before and after CXL and also at various IOPs.

\section{Theory and calculations}

\subsection{Modified Rayleigh-Lamb frequency equation}

Since the air-pulse induced displacement in the cornea is in the order of microns, which is a few orders of magnitude smaller than the thickness of the cornea, we can reasonably utilize the Navier-Cauchy equation that governs the dynamic response of an isotropic, homogenous, Kelvin-Voigt viscoelastic material with small displacement assumption as:

$$
\rho \frac{\partial^{2} \mathbf{u}}{\partial t^{2}}=(\lambda+\mu) \nabla \nabla \cdot \mathbf{u}+\mu \nabla^{2} \mathbf{u}+\eta \nabla \nabla \cdot \frac{\partial \mathbf{u}}{\partial t}+\eta \nabla^{2} \frac{\partial \mathbf{u}}{\partial t}
$$

where $\rho$ is the material density, $\mathbf{u}$ is the displacement vector, $t$ is the time, and $\lambda$ and $\mu$ are the Lamé constants with $\lambda=E v /((1+v)(1-2 v))$ and $\mu=E /(2(1+v))$. Additionally, $E$ is the Young's modulus, $\eta$ is the shear viscosity, and $v$ is the Poisson's ratio. Here, we neglect the volume viscosity of the medium.

By introducing a scalar potential $\varphi$ and a vector potential $\psi$ : 


$$
\mathbf{u}=\nabla \varphi+\nabla \times \boldsymbol{\psi},
$$

equation (1) can be decoupled into the two following wave equations:

$$
\begin{gathered}
\rho \frac{\partial^{2} \varphi}{\partial t^{2}}=(\lambda+2 \mu) \nabla^{2} \varphi+2 \eta \nabla^{2} \frac{\partial \varphi}{\partial t}, \\
\text { and } \rho \frac{\partial^{2} \boldsymbol{\psi}}{\partial t^{2}}=(\lambda+2 \mu) \nabla^{2} \boldsymbol{\psi}+2 \eta \nabla^{2} \frac{\partial \boldsymbol{\psi}}{\partial t} .
\end{gathered}
$$

In the Fourier-Hankel domain and in cylindrical coordinates $(R, \gamma, z)$, equations (3) and (4) can be rewritten and simplified as (Nenadic et al., 2011a; Nenadic et al., 2011b):

$$
\left\{\begin{array}{l}
\frac{\partial^{2} \bar{\varphi} H_{0}}{\partial z^{2}}=\alpha^{2} \bar{\varphi}^{H_{0}} \\
\frac{\partial^{2} \bar{\psi}}{\partial z^{2}}=\beta^{2} \bar{\psi}^{H_{1}}
\end{array}\right.
$$

with

$$
\alpha^{2}=k^{2}-\frac{\omega^{2}}{c_{1}^{2}} \text { and } \beta^{2}=k^{2}-\frac{\omega^{2}}{c_{2}^{2}}
$$

where the axisymmetric problem is considered. Here, $c_{1}=\sqrt{\frac{\lambda+2 \mu^{*}}{\rho}}$ is the compressional wave (P-wave) velocity, $c_{2}=\sqrt{\frac{\mu^{*}}{\rho}}$ is the shear wave (S-wave) velocity, $k=\omega / c_{p}$ is the wave number, $c_{p}$ is the phase velocity, $\mu^{*}=\mu+i \omega \eta$ is the dynamic shear modulus, $i$ is the imaginary number, $\eta$ is the shear viscosity, $\omega=2 \pi f$ is the angular frequency, $f$ is the frequency, and $\bar{\psi}^{H_{1}}=\left(0, \bar{\psi}^{H_{1}}, 0\right)^{T}$. The bar represents the transformation of the function into the Fourier domain, and $H_{x}$ represents the transformation into the Hankel domain where $x$ represents the order of the Hankel function. 
The general solutions for equation (5) are:

$$
\left\{\begin{array}{l}
\bar{\varphi}^{H_{0}}=A \sinh (\alpha z)+B \cosh (\alpha z) \\
\bar{\psi}^{H_{1}}=C \sinh (\beta z)+D \cosh (\beta z)
\end{array}\right.
$$

where $A, B, C$, and $D$ are unknown parameters for any specified $k$ and $\omega$.

The displacement and stress fields in the Fourier-Hankel domain are expressed as:

and

$$
\begin{gathered}
\left(\begin{array}{l}
\bar{u}_{z}^{H_{0}} \\
\bar{u}_{r} H_{1}
\end{array}\right)=\left(\begin{array}{c}
\frac{\partial \bar{\varphi}^{H_{0}}}{\partial z}+k \bar{\psi} H_{1} \\
-k \bar{\varphi}^{H_{0}}-\frac{\partial \bar{\psi}^{H_{1}}}{\partial z}
\end{array}\right), \\
\left(\frac{\bar{\sigma}_{z z}^{H_{0}}}{\bar{\sigma}_{z r} H_{1}}\right)=\left(\begin{array}{c}
\mu^{*}\left(\left(k^{2}+\beta^{2}\right) \bar{\varphi}^{H_{0}}+2 k \frac{\partial \bar{\psi} H_{1}}{\partial z}\right) \\
-\mu^{*}\left(2 k \frac{\partial \bar{\varphi}^{H_{0}}}{\partial z}+\left(k^{2}+\beta^{2}\right) \bar{\psi}^{H_{1}}\right.
\end{array}\right)
\end{gathered}
$$

Based on the equations above, the radial $(r-)$ and vertical $\left(z^{-}\right)$solutions of the displacements and stresses in the Fourier-Hankel domain in the cylindrical coordinate are derived as:

$$
\left\{\begin{array}{l}
\bar{u}_{z}^{H_{0}}=\alpha \cosh (\alpha z) \cdot A+\alpha \sinh (\alpha z) \cdot B+k \sinh (\beta z) \cdot C+k \cosh (\beta z) \cdot D \\
\bar{u}_{r}^{H_{1}}=-k \sinh (\alpha z) \cdot A-k \cosh (\alpha z) \cdot B-\beta \cosh (\beta z) \cdot C-\beta \sinh (\beta z) \cdot D \\
\overline{\sigma_{z z}}{ }^{H_{0}}=\mu^{*}\left(\left(k^{2}+\beta^{2}\right) \sinh (\alpha z) \cdot A+\left(k^{2}+\beta^{2}\right) \cosh (\alpha z) \cdot B+2 k \beta \cosh (\beta z) \cdot C+2 k \beta \sinh (\beta z) \cdot D\right) \\
{\overline{\sigma_{z r}}}^{H_{1}}=-\mu^{*}\left(2 k \alpha \cosh (\alpha z) \cdot A+2 k \alpha \sinh (\alpha z) \cdot B+\left(k^{2}+\beta^{2}\right) \sinh (\beta z) \cdot C+\left(k^{2}+\beta^{2}\right) \cosh (\beta z) \cdot D\right)
\end{array}\right.
$$

The posterior surface of the cornea is in contact with the aqueous humor. Our previous work has shown that the aqueous humor has a significant effect on the elastic wave velocity (Han et al., 2016), and thus, it must be considered when estimating corneal biomechanical properties. The 
governing equation for a fluid without any convection and diffusion has been shown as the wave equation (Nenadic et al., 2011a; Nenadic et al., 2011b):

$$
\frac{\partial^{2} \varphi_{F}}{\partial t^{2}}=c_{F}^{2} \nabla^{2} \varphi_{F},
$$

where $c_{F}$ is the compressional wave speed (speed of sound) in the fluid. In cylindrical coordinates, the displacement vector of the fluid, which includes the radial $(r-)$ and vertical $(z-)$ displacements is:

$$
\left(\begin{array}{l}
u_{r F} \\
u_{z F}
\end{array}\right)=\left(\begin{array}{c}
\frac{\partial \varphi_{F}}{\partial r} \\
\frac{\partial \varphi_{F}}{\partial z}
\end{array}\right)
$$

The stress of the fluid is defined as:

$$
\sigma_{z z F}=\rho_{F} \frac{\partial^{2} \varphi_{F}}{\partial t^{2}} .
$$

In the Fourier-Hankel domain, the displacement vectors and stresses of the fluid are:

$$
\left\{\begin{array}{c}
{\overline{u_{r F}}}^{H_{1}}=-k{\overline{\varphi_{F}}}^{H_{0}}=-k A_{F} e^{\alpha_{F} z} \\
{\overline{u_{z F}}}^{H_{0}}=\frac{\partial \overline{\varphi_{F}}}{\partial z}=\alpha_{F} A_{F} e^{\alpha_{F} z} \\
\overline{\sigma_{z z F}}{ }^{H_{0}}=-\rho_{F} \omega^{2} \overline{\bar{\varphi}_{F}}{ }^{H_{0}}=-\rho_{F} \omega^{2} A_{F} e^{\alpha_{F} z}
\end{array}\right.
$$

with

$$
\alpha_{F}^{2}=k^{2}-\frac{\omega^{2}}{c_{F}^{2}},
$$

and $A_{F}$ is an unknown parameter for any specified $k$ and $\omega$. 
The cornea was approximated as a thin-plate for simplicity. To incorporate the true boundary conditions of the cornea, the model has no stress on the anterior surface and fluid on the posterior surface. Here, the boundary conditions can be modeled as:

$$
\begin{aligned}
& z=d, \quad{\overline{\sigma_{z z}}}^{H_{0}}=\overline{\sigma_{z r}}=0 \\
& z=-d, \quad \overline{u_{z}}=\overline{u_{z F}} H_{0} \\
& \overline{\sigma_{z z}} H_{0}=\overline{\sigma_{z z F}} H_{0}, \overline{\sigma_{z r}} H_{1}=0
\end{aligned}
$$

where $d$ is half of the corneal thickness.

The boundary conditions were modified from the standard Rayleigh-Lamb frequency equation (RLFE) (Achenbach, 2012). Based on the modified boundary conditions (16) and by using formulae (10) and (14), we obtain $\operatorname{det}(\mathrm{M})=0$, where

$$
M=\left[\begin{array}{ccccc}
\left(k^{2}+\beta^{2}\right) \sinh (\alpha d) & 2 k \beta \sinh (\beta d) & \left(k^{2}+\beta^{2}\right) \cosh (\alpha d) & 2 k \beta \cosh (\beta d) & 0 \\
2 k \alpha \cosh (\alpha d) & \left(k^{2}+\beta^{2}\right) \cosh (\beta d) & 2 k \alpha \sinh (\alpha d) & \left(k^{2}+\beta^{2}\right) \sinh (\beta d) & 0 \\
-\left(k^{2}+\beta^{2}\right) \sinh (\alpha d) & -2 k \beta \sinh (\beta d) & \left(k^{2}+\beta^{2}\right) \cosh (\alpha d) & 2 k \beta \cosh (\beta d) & \frac{\rho_{F} \omega^{2}}{\mu^{*}} \\
2 k \alpha \cosh (\alpha d) & \left(k^{2}+\beta^{2}\right) \cosh (\beta d) & -2 k \alpha \sinh (\alpha d) & -\left(k^{2}+\beta^{2}\right) \sinh (\beta d) & 0 \\
\alpha \cosh (\alpha d) & k \cosh (\beta d) & -\alpha \sinh (\alpha d) & -k \sinh (\beta d) & -\alpha_{F}
\end{array}\right] .
$$

Equation (17) is termed as the modified RLFE model (mRLFE), which can be applied to a thin plate structure with no stress on the upper surface and with a fluid-structure interface on the lower surface. The viscoelasticity of the corneas was quantified by an iterative gradient-based error minimization procedure, where the error between analytical solution and the OCE measured dispersion curves was minimized. 


\subsection{Comparison of the $m R L F E$ with finite element method}

The results of the mRLFE analytical solution were compared with finite element (FE) simulations. An FE model was constructed in the ANSYS 14.5 environment (ANSYS Inc., PA, USA). The model was a thin plate with thickness of $1 \mathrm{~mm}$ (similar to the thickness of a porcine

cornea), material density of $1062 \mathrm{~kg} / \mathrm{m}^{3}$ to match the cornea (Kampmeier et al., 2000), and Poisson's ratio of 0.49 due to the near incompressibility of corneal tissue (Bryant and McDonnell, 1996; Han et al., 2015b). The boundary conditions of the model matched those of the cornea with air at the upper surface and fluid at the bottom surface as shown in Figure 1 . The fluid density was the same as water at $1000 \mathrm{~kg} / \mathrm{m}^{3}$, and the speed of sound in water was 1500 $\mathrm{m} / \mathrm{s}$. A perturbation was induced at the center of upper surface of the FE model, which is illustrated by the black arrow in Figure 1. The excitation, $y(t)=A_{0} \sin (2 \pi f t)$, was induced at various frequencies, $f$, where $\omega=2 \pi f, A_{0}=0.01 \mathrm{~mm}$, and the phase velocity of the resulting wave at each frequency was calculated to obtain the dispersion curve.

\section{Methods}

\subsection{Porcine samples}

Fresh porcine eyes ( $\mathrm{n}=4$, Sioux Preme Packing Co., Sioux City, IA, USA) were shipped overnight on ice and kept at $4^{\circ} \mathrm{C}$ in refrigerator. Experiments were conducted within 24 hours of receipt of the eyes. Extraneous tissues such as muscles were removed from the eye-globes, and the samples were allowed to come to room temperature before any measurements were conducted. During the OCE measurements, the eye-globe was positioned in a holder to prevent motion. The IOP was controlled by our previously published artificial closed-loop IOP control 
system (Twa et al., 2014). Briefly, the eye-globe was cannulated with two 23G needles. One needle was connected via tubing to a pressure transducer, and the other needle was connected via tubing to a micro-infusion pump to form the closed-loop artificial IOP control system. The IOP control system was controlled by custom software written in Matlab.

The corneas were then crosslinked by the traditional "Dresden" CXL protocol (Wollensak et al., 2003a). Briefly, the epithelium was removed with a blunt surgical instrument. Topical instillation of a $0.1 \%$ riboflavin solution in $20 \%$ dextran solution was performed every 5 minutes for 30 minutes. After the initial 30 mins, the corneas were irradiated for $30 \mathrm{mins}\left(3 \mathrm{~mW} / \mathrm{cm}^{2}, 365\right.$ $\mathrm{nm})$. The topical installation of the riboflavin solution was performed every 5 minutes during the irradiation as well. Immediately following the CXL treatment, the OCE measurements were repeated.

\subsection{Phase stabilized swept source optical coherence elastography (PhS-SSOCE) system}

The home-built phase stabilized swept source OCE (PhS-SSOCE) system consisted of three main parts: a phase-stabilized swept source OCT (PhS-SSOCT) system (Manapuram et al., 2008, 2009), a focused air-pulse delivery system (Wang et al., 2013), and an artificial IOP control system (Twa et al., 2014). Figure 2 shows a schematic of the experimental setup. The PhSSSOCT system utilized a broadband swept laser source (HSL2000, Santec USA Corp., NJ, USA) with a central wavelength of $\sim 1310 \mathrm{~nm}$, scan range of $\sim 150 \mathrm{~nm}$, and scanning rate of $30 \mathrm{kHz}$. The lateral and axial resolutions in air were approximately $16 \mu \mathrm{m}$ and $11 \mu \mathrm{m}$, respectively. The displacement stability of the system was measured as $\sim 40 \mathrm{~nm}$ in the corneal tissue. Further details of the PhS-SSOCT system can be found in our previous work (Manapuram et al., 2008, 2009). Briefly, the focused air-pulse delivery system had an electronically controlled pneumatic 
solenoid and an air-pulse port with an inner diameter of $\sim 150 \mu \mathrm{m}$. The solenoid was controlled by a home-built electronic controller with an input port for synchronization with the PhS-SSOCT system (Wang and Larin, 2014b; Wang et al., 2013). The air-pulse was expelled out of the airport and had a Gaussian temporal profile with a duration of $\sim 800 \mu$ s. The air pressure on the corneal tissue was $<10 \mathrm{~Pa}$ in all measurements. The air-pulse excitation was approximately 2 mm away from the corneal apex to ensure that the air-pulse induced elastic wave propagated across the cornea apex.

The air-pulse induced elastic wave was detected by successive M-mode scans (the OCT probe beam was held in position and measurements were made in time). By synchronizing the air-pulse excitation with the OCT system frame trigger, the OCT system effectively imaged the same elastic wave (Wang and Larin, 2014b). In this work, $501 \mathrm{M}$-mode scans were taken over a $7 \mathrm{~mm}$ line, which was along the nasal/temporal axis of the cornea. OCE measurements were made at IOPs of 15, 20, 25, and $30 \mathrm{mmHg}$ before and after the CXL treatment and along the same axis to minimize effects of the elastic anisotropy of the cornea (Elsheikh et al., 2008b; Nguyen et al., 2014; Pinsky et al., 2005; Singh et al., 2016b).

\subsection{OCE data processing}

The OCE measured raw unwrapped phase profiles from the surface of the sample, $\phi_{\text {surface }}(t)$ were converted to displacement, $\operatorname{disp}_{\text {surface }}(t)$, by:

$$
\operatorname{disp}_{\text {sufface }}(t)=\frac{\lambda_{0}}{4 \pi n_{\text {air }}} \times \phi_{\text {sufface }}(t)
$$


and the unwrapped temporal phase profiles from within the sample, $\phi_{\text {inside }}(t)$, were converted to displacement, $\operatorname{disp}_{\text {inside }}(t)$, after correcting for the sample surface motion and refractive index mismatch between the sample and the air (Wang and Larin, 2014a) by:

$$
\operatorname{disp}_{\text {inside }}(t)=\frac{\lambda_{0}}{4 \pi n_{\text {cornea }}} \times\left[\phi_{\text {inside }}(t)+\phi_{\text {sufface }}(t) \times \frac{n_{\text {cornea }}-n_{\text {air }}}{n_{\text {air }}}\right] .
$$

The refractive index of the cornea, $n_{\text {cornea, }}$ was assumed constant at 1.376 (Mandell, 1994), and the refractive index of air, $n_{\text {air, was }} 1$.

Traditional techniques requiring multiple excitation frequencies are unnecessary as the airpulse induced elastic wave is composed of multiple frequencies. Hence, spectral decomposition of the elastic wave can provide dispersion curves for use in the mRLFE model. A fast Fourier transform (FFT) was performed on each temporal profile at each position and depth in the cornea. Because it was assumed that the elastic wave propagated along the corneal geometry (Wang and Larin, 2014a), the curvature of the cornea was taken into account when calculating the elastic wave propagation distances, $\Delta r$. The phase shifts obtained by FFT, $\Delta \theta$, at each frequency, $f$, were used to calculate the velocity of the elastic wave at each angular frequency by $c_{\mathrm{p}}=2 \pi f \Delta r / \Delta \theta$ with linear fitting. This was repeated for parallel elastic wave propagation paths that followed the corneal geometry for each depth of the cornea. In this work, the spectral resolution was $\sim 59 \mathrm{~Hz}$ and the data was averaged over the whole depth of the cornea.

The viscoelasticity of the corneas was quantified by a gradient based iterative error minimization process where the error between the mRLFE analytical solution and the OCEmeasured dispersion curves was minimized by incrementally adjusting the elasticity and viscosity in the mRLFE. Representative dispersion curves obtained by OCE and the fitted mRLFE for a typical porcine cornea at $20 \mathrm{mmHg}$ IOP (a) before and (b) after CXL are shown in 
Figure 3 where the error bar represents the depth-wise standard deviation for a given phase velocity.

\section{Results}

\subsection{Validation of the $m R L F E$}

Figure 4 plots the dispersion curves of the thin plate structure as obtained by finite element method (FEM) in comparison to the analytical solution of the mRLFE with a Young's modulus of $60 \mathrm{kPa}$ and no viscosity. Clearly the solutions are very similar showing the accuracy of the mRLFE for thin plate structures with air on the upper surface and fluid below the plate. Additionally, the analytical solution for the standard RLFE is plotted to show the effects of the fluid-structure interface at the posterior surface of the sample. The results show that the velocity for a given frequency is lower in the mRLFE as compared to the standard RLFE.

\subsection{Corneal samples}

From the OCT structural image, the central corneal thickness (CCT) was quantified after physically rescaling the images from optical to physical dimensions by assuming the refractive index of the cornea was 1.376 (Mandell, 1994). At IOPs of 15, 20, 25, and $30 \mathrm{mmHg}$, the average CCTs of the untreated corneas were $0.80 \pm 0.03,0.70 \pm 0.08,0.68 \pm 0.08$, and $0.65 \pm 0.08$ $\mathrm{mm}$, respectively. After CXL the average CCTs of the corneas at 15, 20, 25, and $30 \mathrm{mmHg}$ decreased to $0.50 \pm 0.06,0.47 \pm 0.06,0.45 \pm 0.05$, and $0.42 \pm 0.05 \mathrm{~mm}$, respectively. Figure 5 (a) plots the CCT of the samples where the central bar is the mean, and the individual samples are 
represented by the various shapes. The relative decrease in CCT after CXL was $\sim 37 \%, \sim 34 \%$, $\sim 34 \%$, and $\sim 35 \%$ at the IOPs of $15,20,25$, and $30 \mathrm{mmHg}$, respectively.

Figure 5(b) shows the elasticity values of the corneas as assessed by the mRLFE. The Young's moduli of the untreated corneas at 15, 20, 25, and $30 \mathrm{mmHg}$ were 41.8 $\pm 8.1,70.0 \pm 13.0$, 112.5 \pm 18.0 , and $157 \pm 24.1 \mathrm{kPa}$. After CXL, the stiffness of the corneas increased to $87.3 \pm 9.5$, $130.8 \pm 22.0,184.5 \pm 25.6$, and $281.8 \pm 63.3 \mathrm{kPa}$ at IOPs of $15,20,25$, and $30 \mathrm{mmHg}$. At IOPs of $15,20,25$, and $30 \mathrm{mmHg}$, the relative increase in Young's modulus was $\sim 109 \%, \sim 86 \%, \sim 64 \%$, and $\sim 79 \%$, respectively.

The shear viscosity is plotted in Figure 5(c). Before CXL, the shear viscosities of the corneas at $15,20,25$, and $30 \mathrm{mmHg}$ IOP were $0.7 \pm 0.2,0.6 \pm 0.1,0.6 \pm 0.1$, and $0.4 \pm 0.1 \mathrm{~Pa} \bullet \mathrm{s}$, respectively. The shear viscosities decreased to $0.1 \pm 0.1 \mathrm{~Pa} \bullet$ s after CXL for all IOPs. The change in shear viscosity corresponded to a relative decrease of $\sim 86 \%, \sim 84 \%, \sim 83 \%$, and $\sim 81 \%$ at IOPs of 15 , 20, 25, and $30 \mathrm{mmHg}$.

A summary of the linear regression and Pearson's correlation coefficient (PCC) as a function of IOP for the CCT, Young's modulus, and shear viscosity is shown in Table 1. The asterisk indicates statistical significance $(P<0.05)$ by a two tailed t-test of the correlation. Analysis was performed on the mean data for a given sub-set of data (e.g. regression of the mean CCT of all 4 untreated samples as a function of IOP).

\section{Discussion and conclusion}

In this work we have detailed the development of a modified Rayleigh-Lamb frequency equation for thin plate structures with air at the upper surface and fluid at the bottom surface. 
Validation with FEM showed good agreement with the mRLFE analytical solution, showing the accuracy and reliability of the mRLFE model. We then utilized noncontact OCE measurements in combination with the mRLFE to quantify the viscoelasticity of in situ porcine corneas in the whole eye-globe configuration before and after CXL and at various IOPs. The results showed that the stiffness of the corneas increased linearly as a function of IOP, and CXL increased the stiffness of the corneas while decreasing the shear viscosity (Figure 5).

As expected, the CCT decreased as a function of IOP, which has been repeatedly shown in the past by various techniques (Medeiros and Weinreb, 2006; Orssengo and Pye, 1999; Roberts et al., 2011). This is intuitive as a greater pressure in the anterior chamber of the eye will deform the corneal tissue, decreasing its thickness. Previous work has attempted to correlate corneal stiffness with IOP (Liu and Roberts, 2005; Orssengo and Pye, 1999). However, the interplay between corneal stiffness and IOP may not be entirely separable based on elasticity alone. Our previous work has shown that although two corneal samples may have a similar elasticity due to stiffening by CXL or an elevated IOP, their damping characteristics can be used to differentiate the samples (Li et al., 2014a). The results in this work corroborate with the results shown by Li et al, where CXL decreased the viscosity, which is the primary material parameter responsible for damping. This is a powerful result showing that both the elasticity and viscosity are critical for understanding the dynamics of corneal biomechanical properties due to disease or therapeutic interventions such as CXL, particularly when changes in IOP are involved.

The elasticity range of the cornea in the literature is very large, spanning a few orders of magnitude from $1 \mathrm{kPa}$ to greater than $1 \mathrm{MPa}$ depending on many parameters such as testing conditions, species, and most importantly, measurement technique (Dias et al., 2015; Elsheikh et al., 2008a; Mikula et al., 2014; Wollensak et al., 2003b; Zeng et al., 2001). Our own work 
comparing the elasticity or corneas by strip extensiometry and OCE showed the aforementioned difference in elasticity by a few orders of magnitude (Twa et al., 2014). However, it has been postulated that techniques that induce large strains such as mechanical testing have very large equivalent IOPs of up to a few thousand mmHg (Hoeltzel et al., 1992). This is compounded by the "J" shape of the corneal stress-strain curve (Elsheikh et al., 2008a; Elsheikh and Anderson, 2005), resulting in different elasticities at different strains. Thus, direct comparison to material parameters assessed by different techniques is not directly comparable to our in situ OCE measurements. However, this point highlights one of the main advantages of our noncontact OCE technique because the biomechanical properties of the cornea are measured in its native state. Nevertheless, the stiffness quantified by the mRLFE was similar to our previous work using OCE measurements and/or numerical modeling (Han et al., 2015b; Han et al., 2016; Singh et al., 2016c; Singh et al., 2015). The change in corneal stiffness due to CXL is also quite varied in the literature, ranging from an increase of few hundred percent as measured by strip extensiometry and supersonic shear wave imaging (Cherfan et al., 2013; Nguyen et al., 2012; Wollensak et al., 2003b) to an increase of approximately $50 \%$ as assessed by inflation testing and ultrasound elastography (Kling et al., 2010; Palko et al., 2014). Once again, these studies were conducted under different conditions. More comparably, our own work that showed an approximate 50\% increase in stiffness was obtained on rabbit eyes (Singh et al., 2016b), and strip extensiometry has shown a noticeable difference in stiffness changes between human and rabbit eyes, indicating that structural differences between species may also be a contributing factor to corneal stiffening by CXL (Wollensak et al., 2003b).

Viscosity is another parameter that is crucial for biomechanical characterization of tissues but is not easily measured in solids. In this work, the shear viscosity was quantified by the mRLFE, 
and the results showed a large difference between the untreated and CXL-treated tissues. However, this difference decreased as the IOP increased. The ORA and CorVis induce large displacement amplitudes that may induce large equivalent IOPs due to the large displacement amplitude (Hoeltzel et al., 1992). Hence, this may be the reason the ORA and CorVis have difficulty distinguishing untreated and CXL treated corneas because their biomechanical measurements are thought to be primarily related to the viscous damping ability of the cornea rather than its intrinsic stiffness (Bak-Nielsen et al., 2014; Gkika et al., 2012; Goldich et al., 2009; Greenstein et al., 2012).

Our previous work has shown that the thickness, curvature, and fluid structure interface between the corneal posterior surface and aqueous humor all must be considered for accurate biomechanical assessment of the cornea (Han et al., 2015b; Han et al., 2016). Hence, the RLFE was chosen because it incorporates sample thickness and was subsequently modified to incorporate the fluid structure interface, which reduces the wave velocity by absorbing the wave energy as seen in Figure 4 and our previous work (Han et al., 2015a; Han et al., 2016). However, there are limitations to model based on assumptions about the sample. For example, the cornea was assumed as a flat thin plate, which is obviously not true. Additionally, the mRLFE assumes the sample is isotropic, which is also not strictly true of the cornea (Elsheikh and Alhasso, 2009; Elsheikh et al., 2008b; Nguyen et al., 2014; Pinsky et al., 2005; Singh et al., 2016c). Lastly, the mRLFE assumes the sample is homogeneous but the elasticity of the cornea and stiffening effects of the CXL are not homogeneous depth-wise (Beshtawi et al., 2016; Dias et al., 2015; Palko et al., 2014; Scarcelli et al., 2013; Seifert et al., 2014; Wang and Larin, 2014a). This may be a reason for relatively poor fitting of the mRLFE, particularly in the case of CXL samples as seen in Figure 3(b). Nevertheless, the RLFE and mRLFE perform well as compared to 
mechanical testing (Han et al., 2015a; Han et al., 2015c). We are currently developing a more robust multi-layered wave model that can also take into account the curvature of the cornea for more accurate quantification of corneal biomechanical properties.

Compared with FEM, the mRLFE model is robust and takes dramatically less computational time to quantify corneal biomechanical properties (several hours for FEM as compared to a few minutes for the mRFLE). However, FEM is able to simulate wave propagation in structures of arbitrary shapes, but the presented mRLFE model is only suitable for viscoelasticity quantification of structures like the cornea with specific boundary conditions.

In order to provide in vivo assessments of corneal biomechanical properties, the acquisition time will have to be reduced. Moreover, the multiple excitations required by the presented OCE method may negate any minimizations of discomfort due to the small excitation force. We have recently shown an ultra-fast noncontact OCE technique utilizing a single air-pulse excitation where the measurement was taken in milliseconds with only a single air-pulse (Singh et al., 2015). However, the spatial and temporal resolutions were reduced, but maybe be sufficient if combined with robust noise reduction and post-processing algorithms. We are currently investigating the applicability of this technique in conjunction with the mRLFE for measurements of corneal viscoelasticity in vivo.

In summary, we have detailed the development of a modified Rayleigh-Lamb frequency equation wave model for quantifying the viscoelasticity of the cornea. The model incorporates sample thickness and the fluid structure interface as the bottom surface to simulate the geometry of the cornea. The results showed good agreement with finite element method simulations, showing the accuracy of the mRLFE. The mRLFE was then used to quantify the Young's modulus and shear viscosity of in situ porcine corneas before and after CXL and at various IOPs 
based on noncontact OCE measurements. The results showed that CXL stiffened the corneas by $\sim 85 \%$ and decreased the shear viscosity by $\sim 83 \%$. Moreover, the stiffness of the cornea was linear as a function of IOP before and after CXL. This combination of a robust mechanical model and noncontact OCE measurements may provide critical information for early detection of diseases such as keratoconus and evaluating the efficacy of therapeutic interventions such as CXL.

\section{Acknowledgements}

This work was supported in part by the National Institutes of Health grant numbers: 1R01EY022362, 2R01EY022362, 1R01HL130804 and Department of Defense grant PR150338/GRANT11959628

\section{References}

Achenbach, J., 2012. Wave propagation in elastic solids. Elsevier, Amsterdam.

Aglyamov, S.R., Bouchard, R., Graf, I.M., Emelianov, S., 2012. Breast elasticity imaging, in: Markey, M.K. (Ed.), Physics of Mammographic Imaging. CRC press, Boca Raton, FL, pp. 221237.

Ambrosio, R., Jr., Alonso, R.S., Luz, A., Coca Velarde, L.G., 2006. Corneal-thickness spatial profile and corneal-volume distribution: tomographic indices to detect keratoconus. J Cataract Refract Surg 32, 1851-1859.

Azizi, G., Keller, J.M., Mayo, M.L., Piper, K., Puett, D., Earp, K.M., Malchoff, C.D., 2015. Thyroid Nodules and Shear Wave Elastography: A New Tool in Thyroid Cancer Detection. Ultrasound Med Biol 41, 2855-2865.

Bak-Nielsen, S., Pedersen, I.B., Ivarsen, A., Hjortdal, J., 2014. Dynamic Scheimpflug-based assessment of keratoconus and the effects of corneal cross-linking. J Refract Surg 30, 408-414.

Bao, F., Deng, M., Wang, Q., Huang, J., Yang, J., Whitford, C., Geraghty, B., Yu, A., Elsheikh, A., 2015. Evaluation of the relationship of corneal biomechanical metrics with physical intraocular pressure and central corneal thickness in ex vivo rabbit eye globes. Exp Eye Res 137, 11-17.

Beshtawi, I.M., Akhtar, R., Hillarby, M.C., O'Donnell, C., Zhao, X., Brahma, A., Carley, F., Derby, B., Radhakrishnan, H., 2016. Biomechanical Changes of Collagen Cross-Linking on Human Keratoconic Corneas Using Scanning Acoustic Microscopy. Curr Eye Res 41, 609-615. 
Bryant, M.R., McDonnell, P.J., 1996. Constitutive laws for biomechanical modeling of refractive surgery. J Biomech Eng 118, 473-481.

Cherfan, D., Verter, E.E., Melki, S., Gisel, T.E., Doyle, F.J., Jr., Scarcelli, G., Yun, S.H., Redmond, R.W., Kochevar, I.E., 2013. Collagen cross-linking using rose bengal and green light to increase corneal stiffness. Invest Ophthalmol Vis Sci 54, 3426-3433.

Dias, J., Diakonis, V.F., Lorenzo, M., Gonzalez, F., Porras, K., Douglas, S., Avila, M., Yoo, S.H., Ziebarth, N.M., 2015. Corneal stromal elasticity and viscoelasticity assessed by atomic force microscopy after different cross linking protocols. Exp Eye Res 138, 1-5.

Elsheikh, A., Alhasso, D., 2009. Mechanical anisotropy of porcine cornea and correlation with stromal microstructure. Exp Eye Res 88, 1084-1091.

Elsheikh, A., Alhasso, D., Rama, P., 2008a. Biomechanical properties of human and porcine corneas. Exp Eye Res 86, 783-790.

Elsheikh, A., Anderson, K., 2005. Comparative study of corneal strip extensometry and inflation tests. J R Soc Interface 2, 177-185.

Elsheikh, A., Brown, M., Alhasso, D., Rama, P., Campanelli, M., Garway-Heath, D., 2008b. Experimental assessment of corneal anisotropy. J Refract Surg 24, 178-187.

Faruk, T., Islam, M.K., Arefin, S., Haq, M.Z., 2015. The Journey of Elastography: Background, Current Status, and Future Possibilities in Breast Cancer Diagnosis. Clin Breast Cancer 15, 313324.

Ford, M.R., Dupps, W.J., Jr., Rollins, A.M., Roy, A.S., Hu, Z., 2011. Method for optical coherence elastography of the cornea. J Biomed Opt 16, 016005.

Ford, M.R., Sinha Roy, A., Rollins, A.M., Dupps, W.J., Jr., 2014. Serial biomechanical comparison of edematous, normal, and collagen crosslinked human donor corneas using optical coherence elastography. J Cataract Refract Surg 40, 1041-1047.

Gkika, M., Labiris, G., Giarmoukakis, A., Koutsogianni, A., Kozobolis, V., 2012. Evaluation of corneal hysteresis and corneal resistance factor after corneal cross-linking for keratoconus. Graefes Arch Clin Exp Ophthalmol 250, 565-573.

Goldich, Y., Barkana, Y., Morad, Y., Hartstein, M., Avni, I., Zadok, D., 2009. Can we measure corneal biomechanical changes after collagen cross-linking in eyes with keratoconus?--a pilot study. Cornea 28, 498-502.

Gore, D.M., Watson, M.P., Tuft, S.J., 2014. Permanent visual loss in eyes with keratoconus. Acta Ophthalmol 92, e244-245.

Greenstein, S.A., Fry, K.L., Hersh, P.S., 2012. In vivo biomechanical changes after corneal collagen cross-linking for keratoconus and corneal ectasia: 1-year analysis of a randomized, controlled, clinical trial. Cornea 31, 21-25.

Han, Z., Aglyamov, S.R., Li, J., Singh, M., Wang, S., Vantipalli, S., Wu, C., Liu, C.H., Twa, M.D., Larin, K.V., 2015a. Quantitative assessment of corneal viscoelasticity using optical coherence elastography and a modified Rayleigh-Lamb equation. J Biomed Opt 20, 20501.

Han, Z., Li, J., Singh, M., Aglyamov, S.R., Wu, C., Liu, C.H., Larin, K.V., 2015b. Analysis of the effects of curvature and thickness on elastic wave velocity in cornea-like structures by finite element modeling and optical coherence elastography. Appl Phys Lett 106, 233702.

Han, Z., Li, J., Singh, M., Wu, C., Liu, C.H., Wang, S., Idugboe, R., Raghunathan, R., Sudheendran, N., Aglyamov, S.R., Twa, M.D., Larin, K.V., 2015c. Quantitative methods for reconstructing tissue biomechanical properties in optical coherence elastography: a comparison study. Phys Med Biol 60, 3531-3547. 
Han, Z., Tao, C., Zhou, D., Sun, Y., Zhou, C., Ren, Q., Roberts, C.J., 2014. Air puff induced corneal vibrations: theoretical simulations and clinical observations. J Refract Surg 30, 208-213. Han, Z.L., Li, J.S., Singh, M., Vantipalli, S., Aglyamov, S.R., Wu, C., Liu, C.H., Raghunathan, R., Twa, M.D., Larin, K.V., 2016. Analysis of the effect of the fluid-structure interface on elastic wave velocity in cornea-like structures by OCE and FEM. Laser Phys Lett 13, 035602.

Hedbys, B.O., Mishima, S., 1966. The thickness-hydration relationship of the cornea. Exp Eye Res 5, 221-228.

Hoeltzel, D.A., Altman, P., Buzard, K., Choe, K., 1992. Strip extensiometry for comparison of the mechanical response of bovine, rabbit, and human corneas. J Biomech Eng 114, 202-215.

Huang, D., Swanson, E.A., Lin, C.P., Schuman, J.S., Stinson, W.G., Chang, W., Hee, M.R., Flotte, T., Gregory, K., Puliafito, C.A., et al., 1991. Optical coherence tomography. Science 254, 1178-1181.

Iseli, H.P., Thiel, M.A., Hafezi, F., Kampmeier, J., Seiler, T., 2008. Ultraviolet A/riboflavin corneal cross-linking for infectious keratitis associated with corneal melts. Cornea 27, 590-594.

Kampmeier, J., Radt, B., Birngruber, R., Brinkmann, R., 2000. Thermal and biomechanical parameters of porcine cornea. Cornea 19, 355-363.

Kling, S., Remon, L., Perez-Escudero, A., Merayo-Lloves, J., Marcos, S., 2010. Corneal biomechanical changes after collagen cross-linking from porcine eye inflation experiments. Invest Ophthalmol Vis Sci 51, 3961-3968.

Li, J., Han, Z., Singh, M., Twa, M.D., Larin, K.V., 2014a. Differentiating untreated and crosslinked porcine corneas of the same measured stiffness with optical coherence elastography. J Biomed Opt 19, 110502.

Li, J., Wang, S., Manapuram, R.K., Singh, M., Menodiado, F.M., Aglyamov, S., Emelianov, S., Twa, M.D., Larin, K.V., 2013. Dynamic optical coherence tomography measurements of elastic wave propagation in tissue-mimicking phantoms and mouse cornea in vivo. J Biomed Opt 18, 121503.

Li, J.S., Wang, S., Singh, M., Aglyamov, S., Emelianov, S., Twa, M.D., Larin, K.V., 2014b. Airpulse OCE for assessment of age-related changes in mouse cornea in vivo. Laser Phys Lett 11, 065601.

Liu, J., He, X., 2009. Corneal stiffness affects IOP elevation during rapid volume change in the eye. Invest Ophthalmol Vis Sci 50, 2224-2229.

Liu, J., Roberts, C.J., 2005. Influence of corneal biomechanical properties on intraocular pressure measurement: quantitative analysis. J Cataract Refract Surg 31, 146-155.

Manapuram, R.K., Manne, V.G.R., Larin, K.V., 2008. Development of phase-stabilized sweptsource OCT for the ultrasensitive quantification of microbubbles. Laser Phys 18, 1080-1086.

Manapuram, R.K., Manne, V.G.R., Larin, K.V., 2009. Phase-sensitive swept source optical coherence tomography for imaging and quantifying of microbubbles in clear and scattering media. J Appl Phys 105, 102040.

Mandell, R.B., 1994. Corneal power correction factor for photorefractive keratectomy. J Refract Corneal Surg 10, 125-128.

Mazzotta, C., Traversi, C., Caragiuli, S., Rechichi, M., 2014. Pulsed vs continuous light accelerated corneal collagen crosslinking: in vivo qualitative investigation by confocal microscopy and corneal OCT. Eye (Lond) 28, 1179-1183.

Medeiros, F.A., Weinreb, R.N., 2006. Evaluation of the influence of corneal biomechanical properties on intraocular pressure measurements using the ocular response analyzer. J Glaucoma 15, 364-370. 
Mikula, E., Hollman, K., Chai, D., Jester, J.V., Juhasz, T., 2014. Measurement of corneal elasticity with an acoustic radiation force elasticity microscope. Ultrasound Med Biol 40, 16711679.

Muthupillai, R., Lomas, D.J., Rossman, P.J., Greenleaf, J.F., Manduca, A., Ehman, R.L., 1995. Magnetic resonance elastography by direct visualization of propagating acoustic strain waves. Science 269, 1854-1857.

Nenadic, I.Z., Urban, M.W., Aristizabal, S., Mitchell, S.A., Humphrey, T.C., Greenleaf, J.F., 2011a. On Lamb and Rayleigh wave convergence in viscoelastic tissues. Phys Med Biol 56, 6723-6738.

Nenadic, I.Z., Urban, M.W., Mitchell, S.A., Greenleaf, J.F., 2011b. Lamb wave dispersion ultrasound vibrometry (LDUV) method for quantifying mechanical properties of viscoelastic solids. Phys Med Biol 56, 2245-2264.

Ng, A.L., Chan, T.C., Cheng, A.C., 2016. Conventional versus accelerated corneal collagen cross-linking in the treatment of keratoconus. Clin Experiment Ophthalmol 44, 8-14.

Nguyen, T.M., Aubry, J.F., Fink, M., Bercoff, J., Tanter, M., 2014. In vivo evidence of porcine cornea anisotropy using supersonic shear wave imaging. Invest Ophthalmol Vis Sci 55, 75457552.

Nguyen, T.M., Aubry, J.F., Touboul, D., Fink, M., Gennisson, J.L., Bercoff, J., Tanter, M., 2012. Monitoring of cornea elastic properties changes during UV-A/riboflavin-induced corneal collagen cross-linking using supersonic shear wave imaging: a pilot study. Invest Ophthalmol Vis Sci 53, 5948-5954.

Ophir, J., Cespedes, I., Ponnekanti, H., Yazdi, Y., Li, X., 1991. Elastography: a quantitative method for imaging the elasticity of biological tissues. Ultrason Imaging 13, 111-134.

Orssengo, G.J., Pye, D.C., 1999. Determination of the true intraocular pressure and modulus of elasticity of the human cornea in vivo. Bull Math Biol 61, 551-572.

Ortiz-Toquero, S., Perez, S., Rodriguez, G., de Juan, V., Mayo-Iscar, A., Martin, R., 2015. The influence of the refractive correction on the vision-related quality of life in keratoconus patients. Qual Life Res.

Palko, J.R., Tang, J., Cruz Perez, B., Pan, X., Liu, J., 2014. Spatially heterogeneous corneal mechanical responses before and after riboflavin-ultraviolet-A crosslinking. J Cataract Refract Surg 40, 1021-1031.

Pinsky, P.M., van der Heide, D., Chernyak, D., 2005. Computational modeling of mechanical anisotropy in the cornea and sclera. J Cataract Refract Surg 31, 136-145.

Qu, Y., Ma, T., He, Y., Zhu, J., Dai, C., Yu, M., Huang, S., Lu, F., Shung, K.K., Zhou, Q., Chen, Z., 2016. Acoustic Radiation Force Optical Coherence Elastography of Corneal Tissue. IEEE J Sel Top Quantum Electron 22, 1-7.

Randleman, J.B., Khandelwal, S.S., Hafezi, F., 2015. Corneal cross-linking. Surv Ophthalmol 60, 509-523.

Richoz, O., Mavrakanas, N., Pajic, B., Hafezi, F., 2013. Corneal collagen cross-linking for ectasia after LASIK and photorefractive keratectomy: long-term results. Ophthalmology 120, 1354-1359.

Roberts, C.J., Mahmoud, A.M., Ramos, I., Caldas, D., da Silva, R.S., Ambrósio Jr, R., 2011. Factors influencing corneal deformation and estimation of intraocular pressure. Invest Ophthalmol Vis Sci 52, 4384-4384. 
Rosenblat, E., Hersh, P.S., 2016. Intraoperative corneal thickness change and clinical outcomes after corneal collagen crosslinking: Standard crosslinking versus hypotonic riboflavin. J Cataract Refract Surg 42, 596-605.

Ruberti, J.W., Roy, A.S., Roberts, C.J., 2011. Corneal biomechanics and biomaterials. Annu Rev Biomed Eng 13, 269-295.

Sahebjada, S., Fenwick, E.K., Xie, J., Snibson, G.R., Daniell, M.D., Baird, P.N., 2014. Impact of keratoconus in the better eye and the worse eye on vision-related quality of life. Invest Ophthalmol Vis Sci 55, 412-416.

Scarcelli, G., Kling, S., Quijano, E., Pineda, R., Marcos, S., Yun, S.H., 2013. Brillouin microscopy of collagen crosslinking: noncontact depth-dependent analysis of corneal elastic modulus. Invest Ophthalmol Vis Sci 54, 1418-1425.

Scarcelli, G., Pineda, R., Yun, S.H., 2012. Brillouin optical microscopy for corneal biomechanics. Invest Ophthalmol Vis Sci 53, 185-190.

Schmitt, J., 1998. OCT elastography: imaging microscopic deformation and strain of tissue. Opt Express 3, 199-211.

Seifert, J., Hammer, C.M., Rheinlaender, J., Sel, S., Scholz, M., Paulsen, F., Schaffer, T.E., 2014. Distribution of Young's modulus in porcine corneas after riboflavin/UVA-induced collagen cross-linking as measured by atomic force microscopy. PLoS One 9, e88186.

Seiler, T.G., Fischinger, I., Koller, T., Zapp, D., Frueh, B.E., Seiler, T., 2016. Customized Corneal Cross-linking: One-Year Results. Am J Ophthalmol 166, 14-21.

Singh, M., Li, J., Han, Z., Vantipalli, S., Liu, C.H., Wu, C., Raghunathan, R., Aglyamov, S.R., Twa, M.D., Larin, K.V., 2016a. Evaluating the Effects of Riboflavin/UV-A and RoseBengal/Green Light Cross-Linking of the Rabbit Cornea by Noncontact Optical Coherence Elastography. Invest Ophthalmol Vis Sci 57, OCT112-120.

Singh, M., Li, J., Han, Z., Wu, C., Aglyamov, S.R., Twa, M.D., Larin, K.V., $2016 b$. Investigating Elastic Anisotropy of the Porcine Cornea as a Function of Intraocular Pressure With Optical Coherence Elastography. J Refract Surg 32, 562-567.

Singh, M., Li, J., Vantipalli, S., Wang, S., Han, Z., Nair, A., Aglyamov, S.R., Twa, M.D., Larin, K.V., 2016c. Noncontact elastic wave imaging optical coherence elastography for evaluating changes in corneal elasticity due to crosslinking. IEEE J Sel Top Quantum Electron 22, 1-11.

Singh, M., Wu, C., Liu, C.H., Li, J., Schill, A., Nair, A., Larin, K.V., 2015. Phase-sensitive optical coherence elastography at 1.5 million A-Lines per second. Opt Lett 40, 2588-2591.

Sticker, M., Hitzenberger, C.K., Leitgeb, R., Fercher, A.F., 2001. Quantitative differential phase measurement and imaging in transparent and turbid media by optical coherence tomography. Opt Lett 26, 518-520.

Tao, C., Han, Z., Sun, Y., Zhou, C., Roberts, C., Zhou, D., Ren, Q., 2013. Corneal hysteresis with intraocular pressure of a wide range: a test on porcine eyes. J Refract Surg 29, 850-854.

Twa, M.D., Li, J., Vantipalli, S., Singh, M., Aglyamov, S., Emelianov, S., Larin, K.V., 2014. Spatial characterization of corneal biomechanical properties with optical coherence elastography after UV cross-linking. Biomed Opt Express 5, 1419-1427.

Venkatesh, S.K., Yin, M., Ehman, R.L., 2013. Magnetic resonance elastography of liver: technique, analysis, and clinical applications. J Magn Reson Imaging 37, 544-555.

Wang, S., Larin, K.V., 2014a. Noncontact depth-resolved micro-scale optical coherence elastography of the cornea. Biomed Opt Express 5, 3807-3821.

Wang, S., Larin, K.V., 2014b. Shear wave imaging optical coherence tomography (SWI-OCT) for ocular tissue biomechanics. Opt Lett 39, 41-44. 
Wang, S., Larin, K.V., 2015. Optical coherence elastography for tissue characterization: a review. J Biophotonics 8, 279-302.

Wang, S., Larin, K.V., Li, J.S., Vantipalli, S., Manapuram, R.K., Aglyamov, S., Emelianov, S., Twa, M.D., 2013. A focused air-pulse system for optical-coherence-tomography-based measurements of tissue elasticity. Laser Phys Lett 10, 075605.

Wollensak, G., Spoerl, E., Seiler, T., 2003a. Riboflavin/ultraviolet-a-induced collagen crosslinking for the treatment of keratoconus. Am J Ophthalmol 135, 620-627.

Wollensak, G., Spoerl, E., Seiler, T., 2003b. Stress-strain measurements of human and porcine corneas after riboflavin-ultraviolet-A-induced cross-linking. J Cataract Refract Surg 29, 17801785.

Zeng, Y.J., Yang, J., Huang, K., Lee, Z.H., Lee, X.Y., 2001. A comparison of biomechanical properties between human and porcine cornea. J Biomech 34, 533-537.

Zhang, X., Tao, X.C., Zhang, J., Li, Z.W., Xu, Y.Y., Wang, Y.M., Zhang, C.X., Mu, G.Y., 2015. A review of collagen cross-linking in cornea and sclera. J Ophthalmol 2015, 289467. 


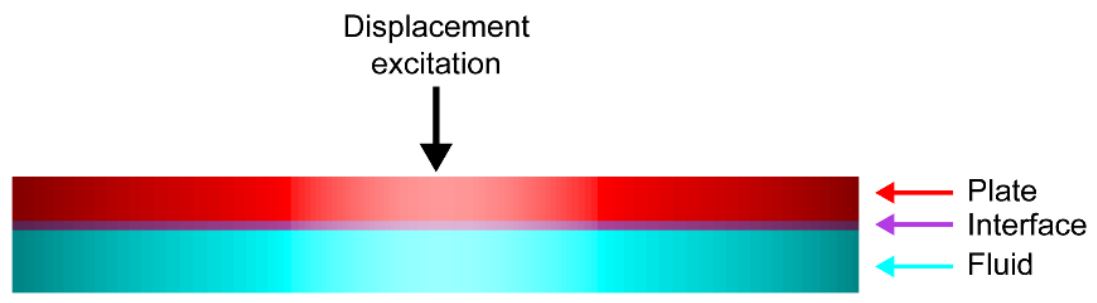

(a)

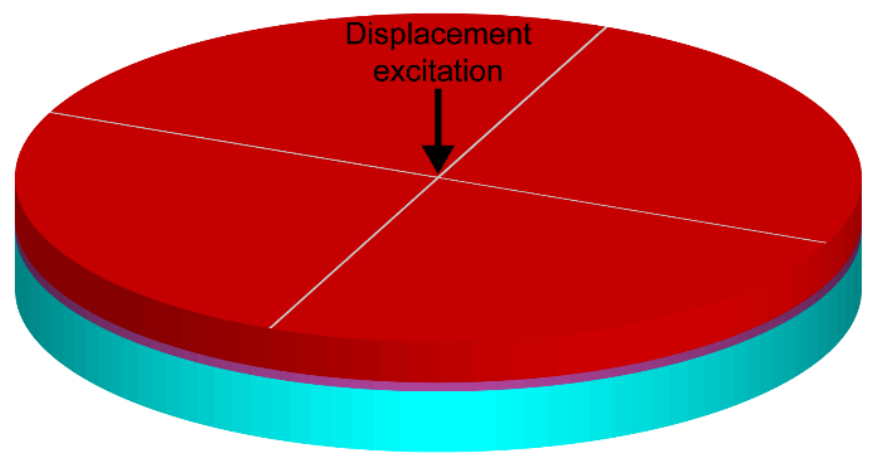

(b)

Figure 1. (a) 2D and (b) 3D views of the thin plate FE model with air on the upper surface and a fluid structure interface at the bottom surface. The displacement excitation was at the center of the upper surface of the plate as indicated. 


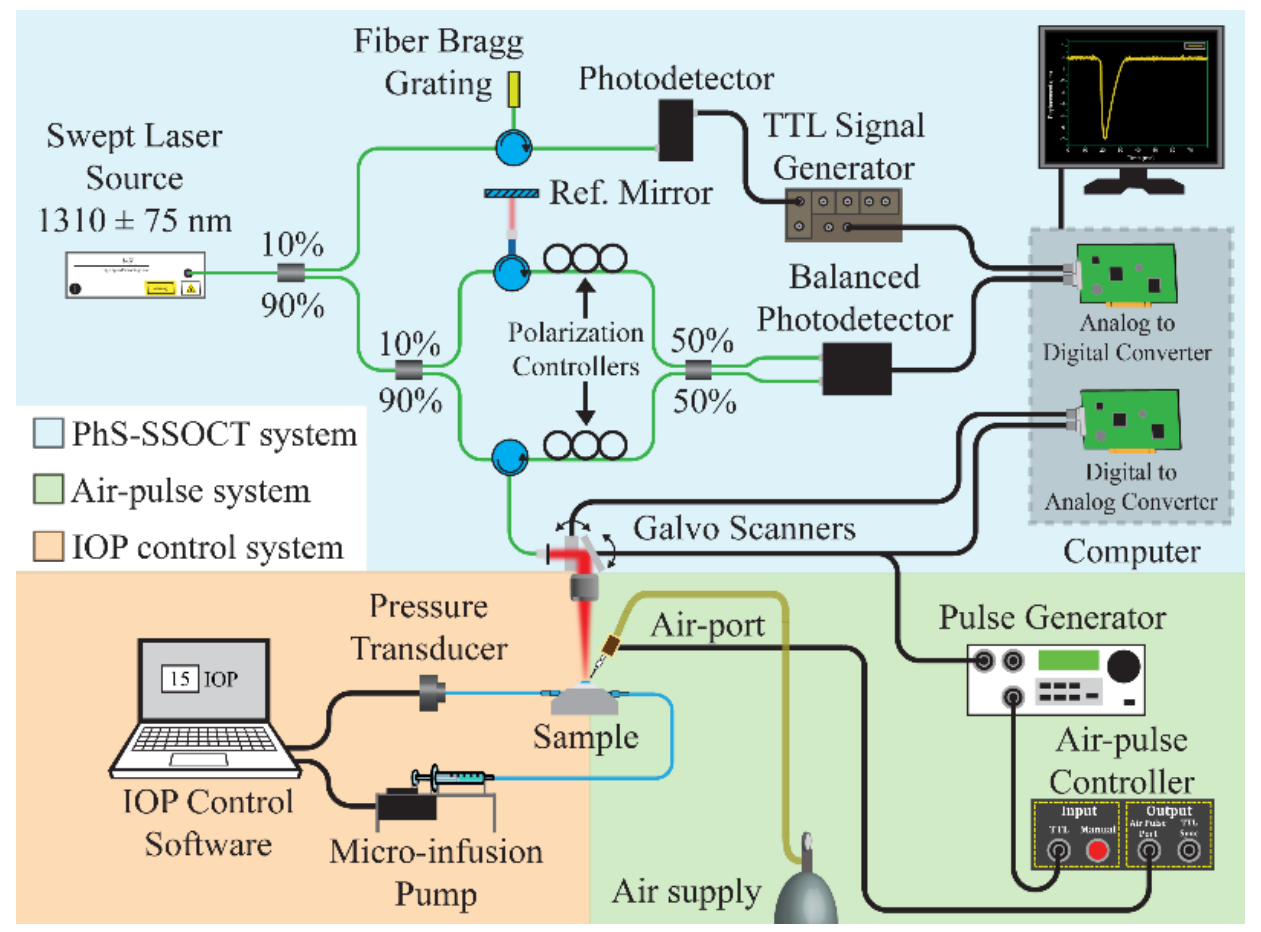

Figure 2. Schematic of the OCE experimental setup. 


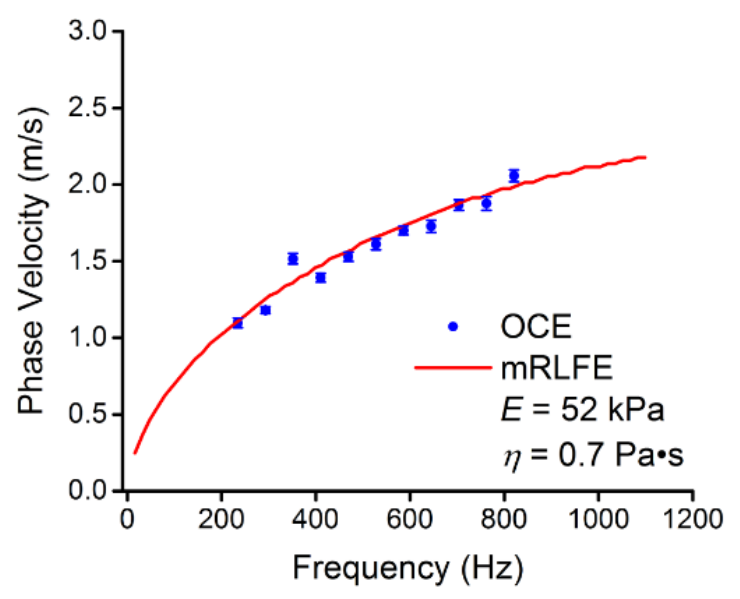

(a)

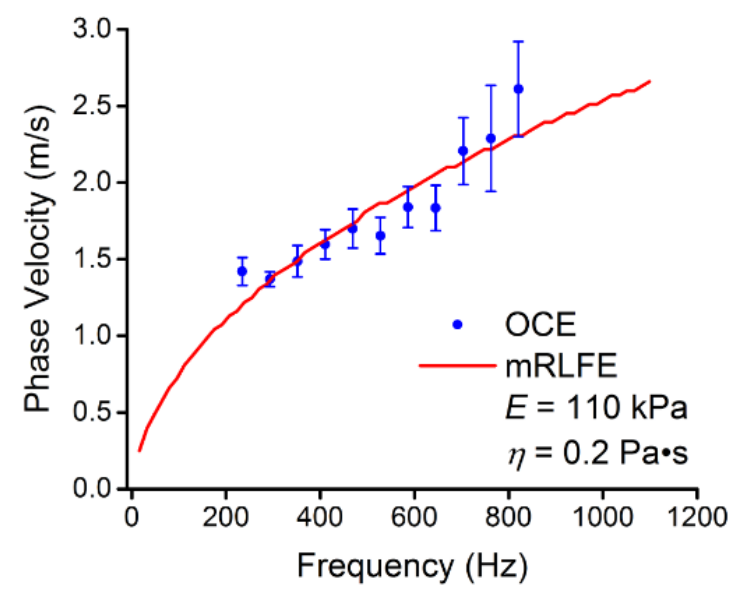

(b)

Figure 3. Dispersion curves of a typical porcine corneal sample at $20 \mathrm{mmHg}$ as obtained by OCE and the fitted mRLFE analytical solution (a) before and (b) after CXL. 


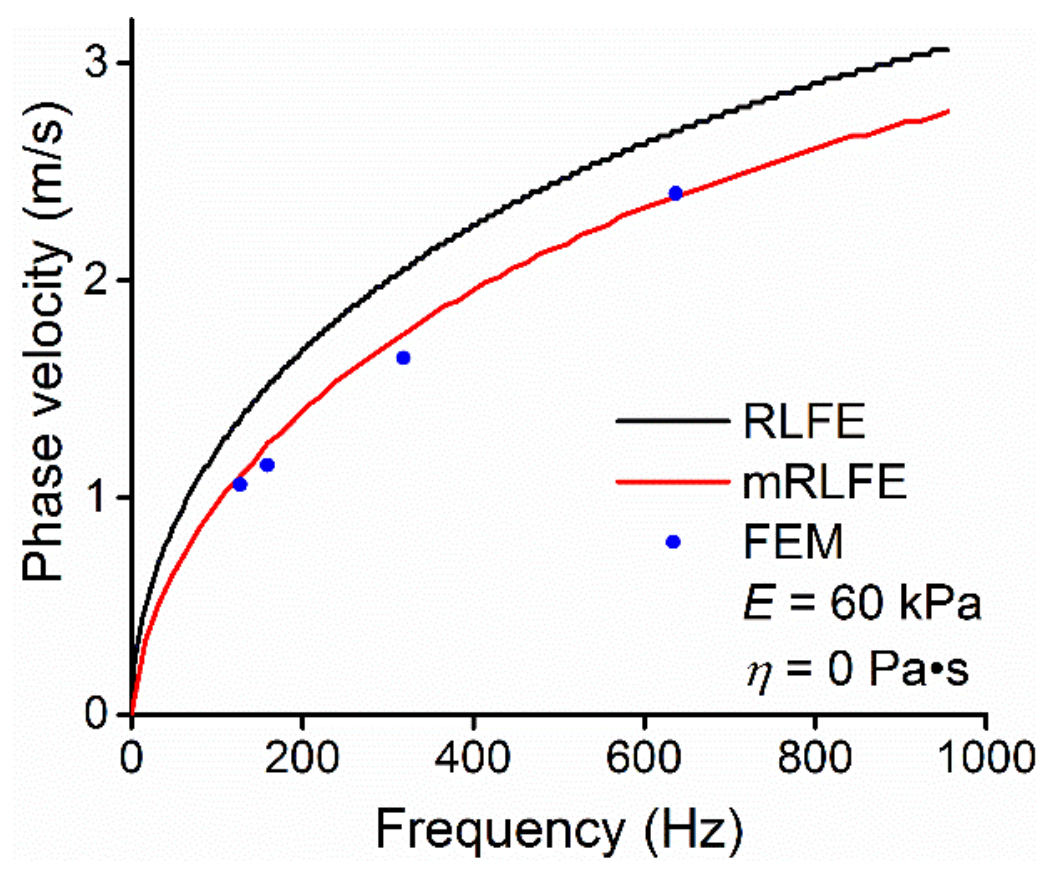

Figure 4: Comparison of the mRLFE analytical solutions and FEM of a thin plate structure with air at the upper surface and fluid at the bottom surface and the standard RFLE. Young's modulus was set to $60 \mathrm{kPa}$ and the viscosity was $0 \mathrm{~Pa} \bullet$ in all three cases. 


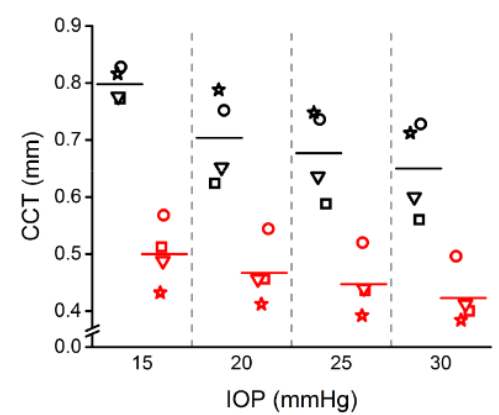

(a)

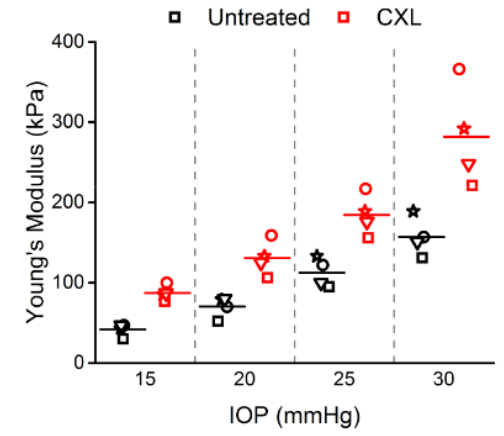

(b)

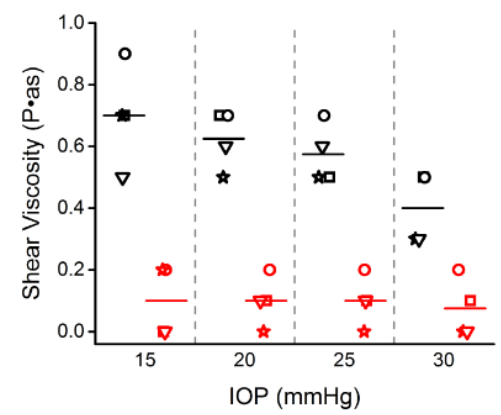

(c)

Figure 5. (a) Central Corneal Thickness (CCT) of untreated and CXL porcine corneal samples at the various IOPs after rescaling to physical dimensions. (b) Young's modulus and (c) shear viscosity of the samples. The individual samples are represented by the various shapes. 
Table 1. Linear regression and PCC for the CCT, Young's modulus, and shear viscosity as a function of IOP.

The asterisk denotes statistical significance $(P<0.05)$ by a two tailed t-test of the correlation with IOP.

\begin{tabular}{rcccc} 
& \multicolumn{2}{c}{ Slope } & \multicolumn{2}{c}{ PCC } \\
\cline { 2 - 5 } Parameter & Untreated & CXL & Untreated & CXL \\
\hline CCT & $-10.9 \mu \mathrm{m} / \mathrm{mmHg}$ & $-5.0 \mu \mathrm{m} / \mathrm{mmHg}$ & $-0.972^{*}$ & $-0.995^{*}$ \\
& & $10.3 \mathrm{kPa} / \mathrm{mmHg}$ & $0.994^{*}$ & $0.987^{*}$ \\
Young's modulus & $7.3 \mathrm{kPa} / \mathrm{mmHg}$ & $-2 \mathrm{mPa} \bullet \mathrm{s} / \mathrm{mmHg}$ & -0.948 & -0.752
\end{tabular}



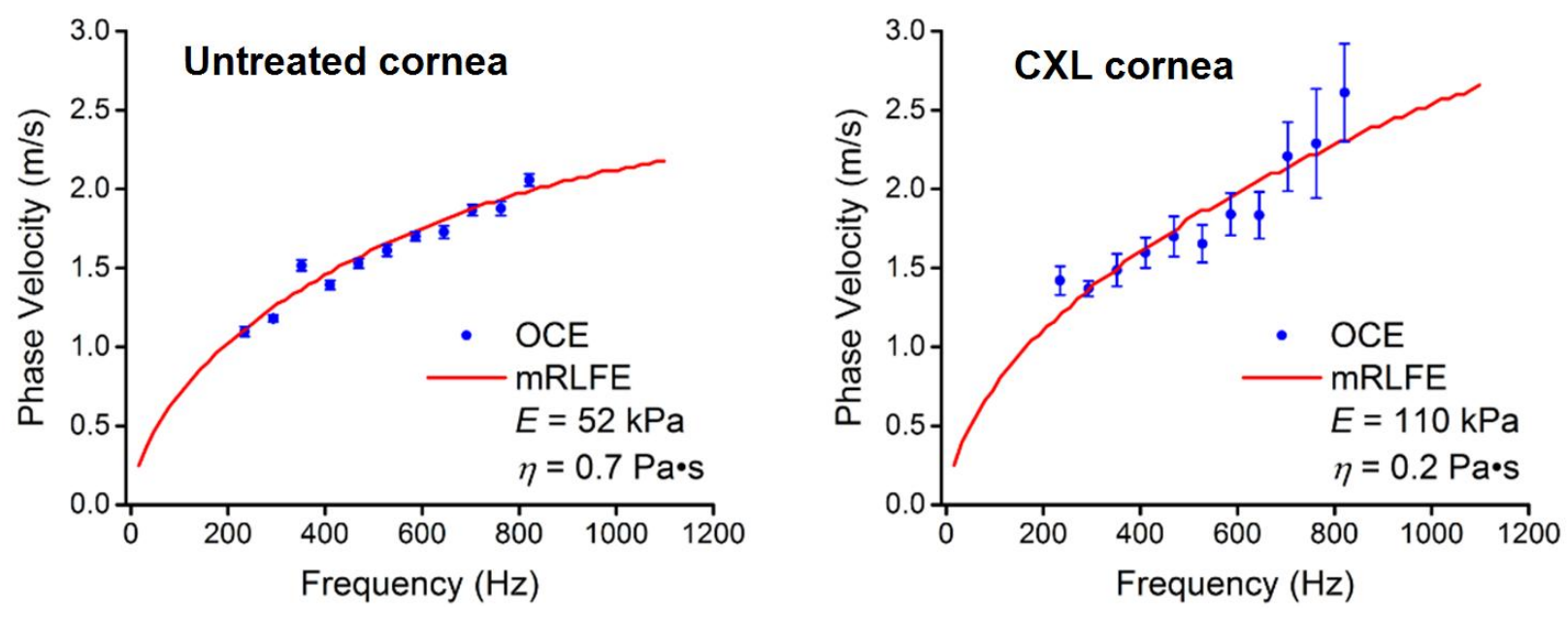